\title{
A case of long-term survival of metastatic desmoplastic small round cell tumor treated with multimodal therapy
}

\author{
TSUBASA WATANABE ${ }^{1}$, SHIN'ICHI MIYAMOTO $^{1}$, KOJI KITAGORI $^{1}$, \\ TAKAHIRO HORIMATSU ${ }^{1}$, SHUKO MORITA ${ }^{1}$, YOKO MASHIMO ${ }^{1}$, \\ YASUMASA EZOE ${ }^{2}$, MANABU MUTO ${ }^{1}$ and TSUTOMU CHIBA ${ }^{1}$ \\ Departments of ${ }^{1}$ Gastroenterology and Hepatology, and ${ }^{2}$ Multidisciplinary Cancer Treatment, \\ Graduate School of Medicine, Kyoto University, Sakyo-ku, Kyoto 606-8507, Japan
}

Received June 8, 2011; Accepted October 12, 2011

DOI: $10.3892 / 01.2011 .457$

\begin{abstract}
Desmoplastic small round cell tumor (DSRCT) is a rare, aggressive and malignant tumor that predominantly affects young males. No standard therapy is currently available for patients with DSRCT and the prognosis remains extremely poor. In this study, we report a thought-provoking DSRCT case. A 24-year-old male was admitted to our hospital with a chief complaint of hematemesis. Computed tomography revealed a retrovesical mass with a splenic hilar tumor, multiple lung and liver tumors and marked lymph node swellings. The source of hematemesis was gastric varices caused by the compression of the splenic vein by a splenic hilar tumor. The patient was provided with a histological diagnosis of DSRCT based on needle biopsy from the liver tumors and the pelvic mass was thought to be the primary lesion. This is a long-term survival case of metastatic DSRCT treated with multimodal therapy including 15 courses of multiagent chemotherapy, radiation therapy for the hepatic portal region using 42.5 Gy, and four instances of therapeutic endoscopy. The prolonged progression-free survival period (15 months) obtained following chemotherapy suggests the chemosensitive feature of the disease. We used a modified P6 regimen (cyclophosphamide, pirarubicin, vincristine, ifosfamide and etoposide) and a modified PAVEP regimen (cyclophosphamide, pirarubicin, etoposide and cisplatin) to decrease severe adverse events and to improve the completion rate of chemotherapy. DSRCT is an aggressive but chemo-sensitive disease, and continuous chemotherapy using an appropriate regimen with possible supportive care is essential for long-term survival. This case report may represent a treatment option for this rare disease.
\end{abstract}

Correspondence to: $\mathrm{Dr}$ Shin'ichi Miyamoto, Department of Gastroenterology and Hepatology, Graduate School of Medicine, Kyoto University, 54 Shogoin-Kawaharacho, Sakyo-ku, Kyoto 606-8507, Japan

E-mail: shmiyamo@kuhp.kyoto-u.ac.jp

Key words: desmoplastic small round cell tumor, multimodal therapy, P6 regimen, PAVEP regimen

\section{Introduction}

Desmoplastic small round cell tumor (DSRCT) is a rare, aggressive, malignant tumor that predominantly affects young males at a median age of 19 years (range 7-58) and with a male-to-female ratio ranging from 5:1 to 10:1 $(1,2)$. DSRCT is a member of the small round blue cell tumor family, which includes small-cell carcinoma, Merkel cell carcinoma, synovial sarcoma, Ewing's sarcoma/primitive neuroectodermal tumor, neuroblastoma, lymphoma, rhabdomyosarcoma and DSRCT (3). No standard therapy is currently available for patients with DSRCT and the prognosis of DSRCT remains extremely poor (2). In this study, we report a case of longterm survival of metastatic DSRCT treated with multimodal therapy, including multiagent chemotherapy, radiation therapy and therapeutic endoscopy.

\section{Case history}

A 24-year-old male was admitted to our hospital with a chief complaint of hematemesis. The patient had neither significant medical history nor family history. Physical examination revealed only that the patient was anemic. Laboratory examination was as follows: hemoglobin, $11.4 \mathrm{~g} / \mathrm{dl}$ (normal range 13.5-16.7 g/dl); white blood cell count, 6,200/dl (normal range $2,900-8,900 / \mathrm{dl}$ ); platelet count, $13.2 \times 10^{10} / \mathrm{dl}$ (normal range, $15.9-38.9 \times 10^{10} / \mathrm{dl}$ ); C-reactive protein, $0.1 \mathrm{mg} / \mathrm{dl}$ (normal value $\leq 0.2 \mathrm{mg} / \mathrm{dl}$ ); aspartate aminotransferase, $52 \mathrm{IU} / 1$ (normal range 13-33 IU/l); alanine aminotransferase, $99 \mathrm{IU} / 1$ (normal range 8-42 IU/l); alkaline phosphatase, $588 \mathrm{IU} / 1$ (normal range 115-359 IU/l); $\gamma$-glutamyl transpeptidase, $380 \mathrm{IU} / 1$ (normal range 9-54 IU/l); and total bilirubin, $0.7 \mathrm{mg} / \mathrm{dl}$ (normal range $0.3-1.3 \mathrm{mg} / \mathrm{dl})$. Renal function tests were normal.

Endoscopy showed oozing bleeding from varicose veins located on the greater curvature of the upper gastric body (Fig. 1A). Spontaneous hemostasis was obtained. Computed tomography (CT) demonstrated that compression of the splenic vein by the splenic hilar tumor appeared to cause the gastric varices (Fig. 1B). CT revealed the presence of a well-enhanced, bulky and lobulated mass on the pelvic floor (Fig. 1C) with a splenic hilar tumor, multiple liver and lung tumors, and marked lymph node swellings (particularly in 

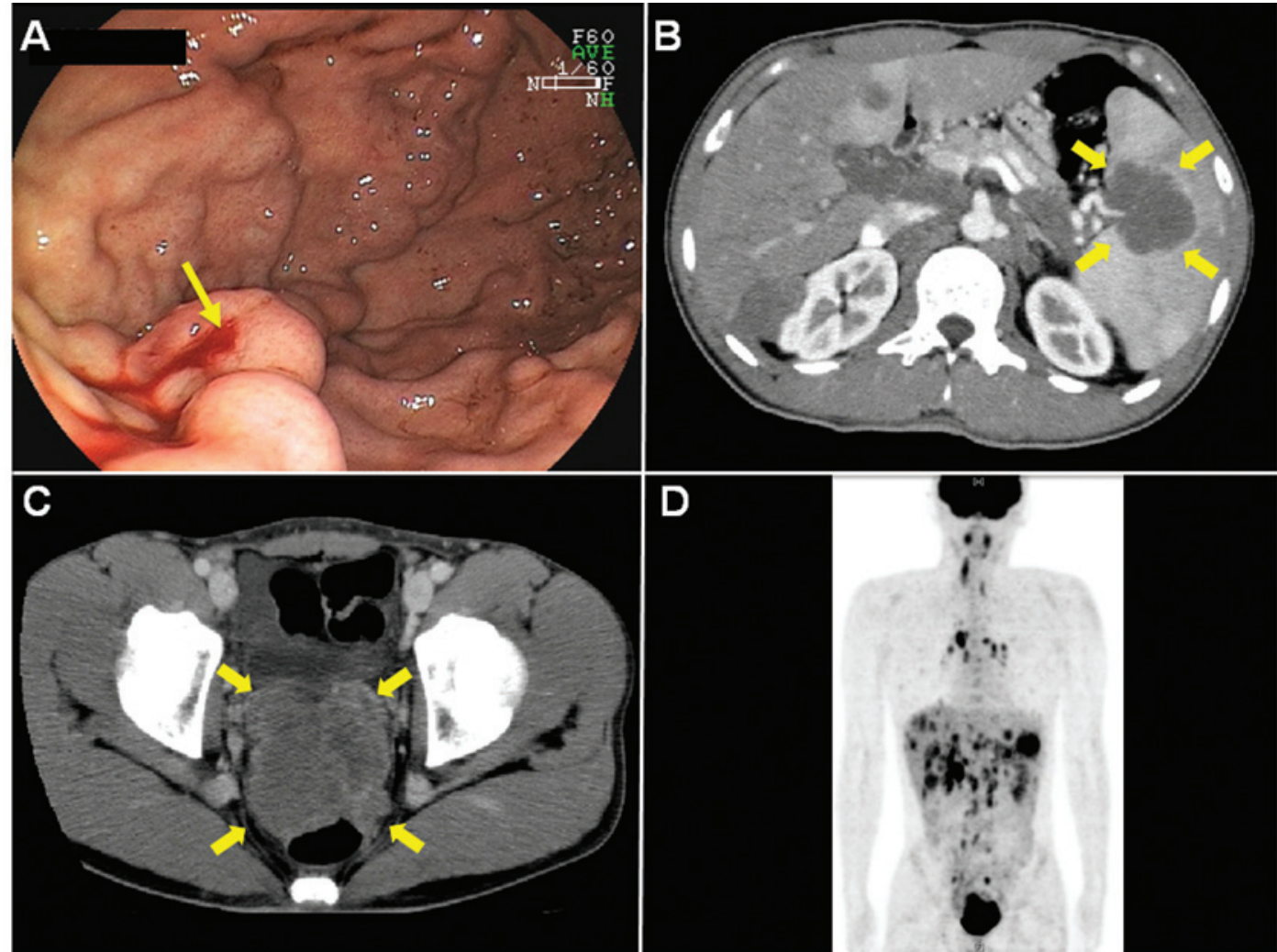

Figure 1. Endoscopic and radiological findings on admission. Endoscopy showed oozing bleeding from gastric varices (A, arrow shows bleeding point). Compression of the splenic vein by the splenic hilar tumor appeared to cause the gastric varices (B, arrows). A well-enhanced, bulky and lobulated tumor located on the pelvic floor was thought to be the primary lesion (C, arrows). ${ }^{18} \mathrm{~F}$-fluorodeoxyglucose (FDG) positron emission tomography revealed intense FDG uptake in the same tumors detected using computed tomography (D, a primary lesion, a splenic hilar tumor and multiple liver, lung and lymph node lesions).
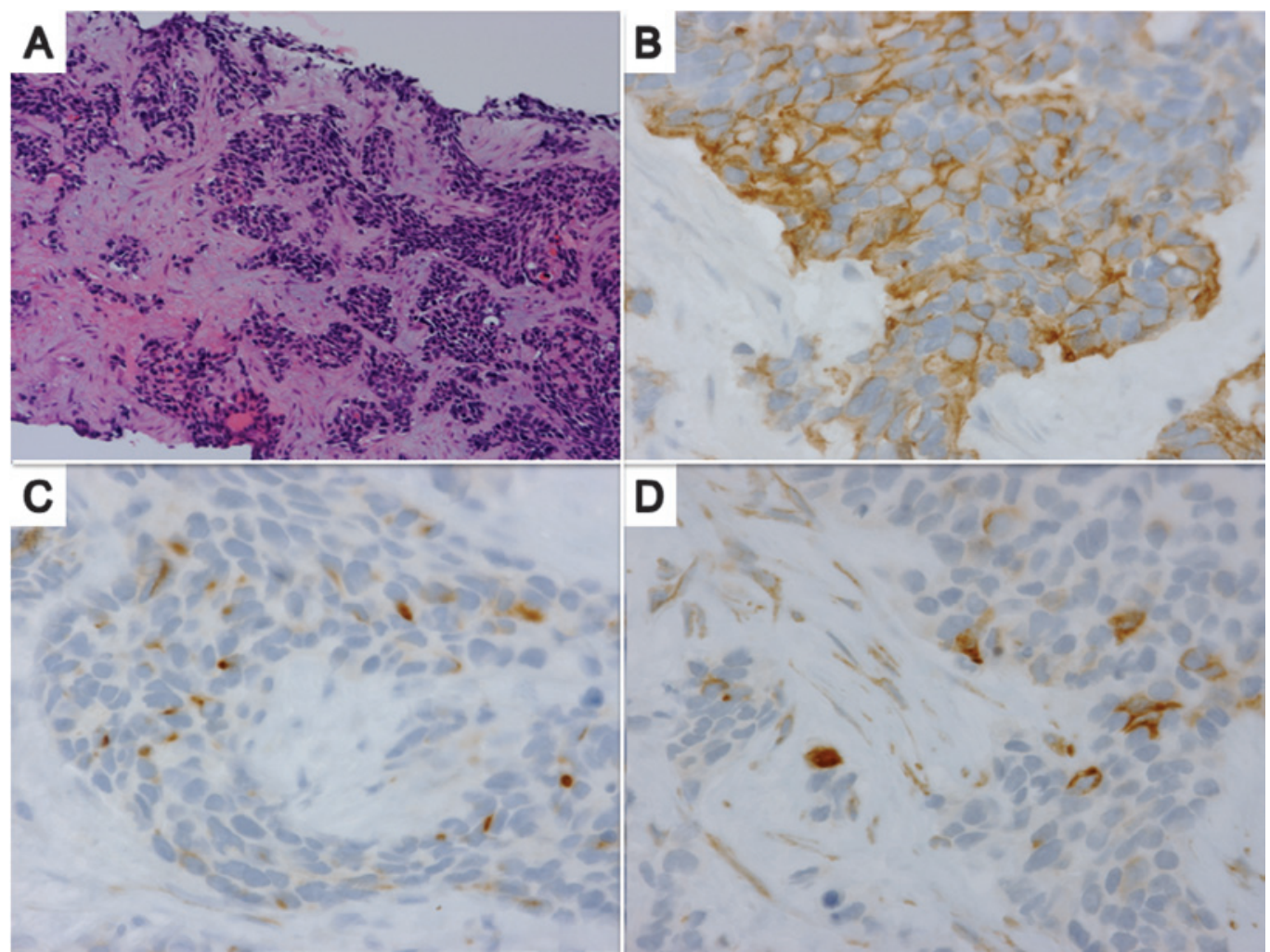

Figure 2. Representative pathological features of a needle biopsy specimen from the liver tumor. Hematoxylin and eosin staining showed poorly differentiated tumor cells with variable size and shape, composed of nests of small round cells and surrounded by a prominent desmoplastic stroma (A, magnification, x100). Immunohistochemical staining was positive for (B) cytokeratin (magnification, $\mathrm{x} 400$ ), (C) desmin (magnification, $\mathrm{x} 400$ ) and (D) Wilms' tumor 1 protein (magnification, x400). 


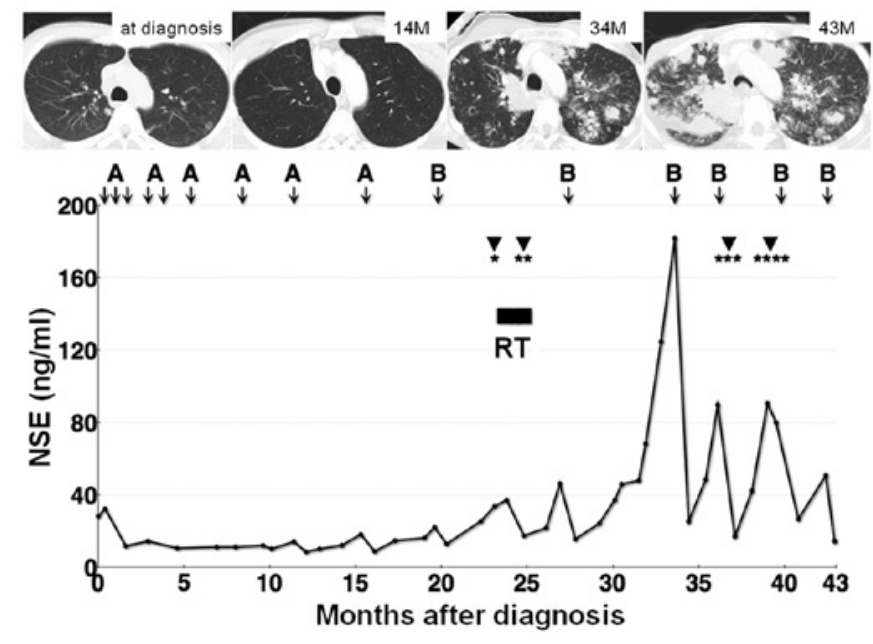

Figure 3. Clinical course of this case. Serum NSE level correlated well with clinical response. The patient received (A) nine courses of a modified P6 regimen and (B) six courses of a modified PAVEP regimen. Obstructive jaundice caused by portal lymphadenopathy was treated successfully by repeated endoscopic biliary drainage and radiation therapy (RT) to the hepatic portal region using $42.5 \mathrm{~Gy}$, at $1.8 \mathrm{~Gy}$ per fraction. Massive hematemesis caused by active bleeding from the varicose vein was treated successfully by endoscopic hemostasis. The patient succumbed to acute pulmonary failure caused by progressive pulmonary metastases 43 months following diagnosis. Time-series CT images of pulmonary metastases are shown in parallel in the upper column (M, months after diagnosis). *Endoscopic biliary drainage using a plastic stent; ${ }^{* *}$ endoscopic biliary drainage using a metal stent; ${ }^{* * *}$ endoscopic hemostasis using metal clips for the active bleeding from a varicose vein; ${ }^{* * * *}$ the metal stent obstruction caused by tumor ingrowth was relieved by inserting a plastic stent into the prior metal stent. NSE, neuron-specific enolase.

the hepatic portal region). ${ }^{18} \mathrm{~F}$-fluorodeoxyglucose positron emission tomography showed multiple accumulation of a glucose analog in the same lesions detected using CT (Fig. 1D).

A needle biopsy specimen from the liver tumor revealed the presence of a poorly differentiated tumor with a variable size and shape, composed of nests of small round cells surrounded by a prominent desmoplastic stroma (Fig. 2A). Immunohistochemically, tumor cells coexpressed an epithelial marker (cytokeratin, Fig. 2B), a mesenchymal marker (desmin, Fig. 2C) and the Wilms' tumor 1 protein (Fig. 2D). Chromogranin, cluster of differentiation antigen (CD) 99 and CD56 were negative. From these findings, the patient was provided with a definite diagnosis of pelvic cavity-origin DSRCT with multiple-organ metastases $(4,5)$.

Theclinicalcourse of this case is shown inFig. 3. The patient was initially treated with multiagent chemotherapy using cyclophosphamide, pirarubicin, vincristine, ifosfamide and etoposide, according to the Ewing's sarcoma protocol, which is a modified protocol of the P6 regimen using pirarubicin instead of doxorubicin (modified P6 regimen) $(2,6)$. During each course of this chemotherapy, the patient suffered from severe nausea and vomiting. The patient required frequent blood transfusions and continuous use of granulocyte colonystimulating factor due to severe bone marrow suppression. The multiple pulmonary metastases were almost eradicated following four courses of the modified P6 regimen and the patient reached 15 months of progression-free survival after the application of this modified P6 regimen (Fig. 3). After
Table I. Chemotherapy regimens reported previously and used in this case.

Dose Day

\section{P6 (6) \\ Courses 1, 2, 3 and 6 \\ Cyclophosphamide \\ Doxorubicin \\ Vincristine \\ Courses 4, 5 and 7 \\ Ifosfamide \\ Etoposide \\ PAVEP (7) \\ Doxorubicin \\ Cyclophosphamide \\ Etoposide \\ Cisplatin}

$2.1 \mathrm{~g} / \mathrm{m}^{2}$
$25 \mathrm{mg} / \mathrm{m}^{2}$
$0.67 \mathrm{mg} / \mathrm{m}^{2}$

$1.8 \mathrm{~g} / \mathrm{m}^{2}$

$100 \mathrm{mg} / \mathrm{m}^{2}$

$40 \mathrm{mg} / \mathrm{m}^{2}$

$300 \mathrm{mg} / \mathrm{m}^{2}$

$75 \mathrm{mg} / \mathrm{m}^{2}$

$100 \mathrm{mg} / \mathrm{m}^{2}$

Modified P6

Courses 1, 3, 5 and 7

Cyclophosphamide

Pirarubicin

Vincristine

Courses 2, 4 and 6

Ifosfamide

Etoposide

$$
2 \mathrm{~g} / \mathrm{m}^{2}
$$

$20 \mathrm{mg} / \mathrm{m}^{2}$

1-3

$2 \mathrm{mg} / \mathrm{m}^{2}$

\section{$2.5 \mathrm{~g} / \mathrm{m}^{2}$}

$100 \mathrm{mg} / \mathrm{m}^{2}$

\section{Modified PAVEP \\ Pirarubicin \\ Cyclophosphamide \\ Etoposide \\ Cisplatin}

$40 \mathrm{mg} / \mathrm{m}^{2}$

$450 \mathrm{mg} / \mathrm{m}^{2}$

$110 \mathrm{mg} / \mathrm{m}^{2}$

1-2

Courses started after confirmation that the neutrophil count reached $500 / \mu 1$ and that the platelet count was $>10,000 / \mu 1$.

the nine courses of treatment, second-line chemotherapy based on the PAVEP regimen (doxorubicin, cyclophosphamide, etoposide and cisplatin) (7) was introduced due to disease progression. To reduce adverse events, we modified the PAVEP regimen by using pirarubicin instead of doxorubicin and shortening the period of the regimen from five to two days (modified PAVEP regimen). The P6, modified P6, PAVEP and modified PAVEP regimens are shown in Table I.

Obstructive jaundice caused by portal lymphadenopathy developed 23 months following diagnosis. Endoscopic biliary drainage using a plastic stent was successfully performed. However, stent obstruction occurred two months after the initial placement of the plastic stent. Subsequently, we removed the stent and inserted a metal stent, which was followed by irradiation of the hepatic portal region using a total dose of $42.5 \mathrm{~Gy}$, at $1.8 \mathrm{~Gy}$ per fraction. The patient had massive hematemesis 37 months following diagnosis caused by the active bleeding from a known varicose vein and endoscopic hemostasis using 
metal clips was obtained successfully. Furthermore, metal stent obstruction caused by tumor ingrowth occurred 39 months following diagnosis, which was relieved by inserting a plastic stent into the metal stent placed previously. After 15 courses of chemotherapy, radiation therapy and four instances of endoscopic therapy (Fig. 3), the patient experienced sudden respiratory arrest caused by bronchial obstruction by the parabronchial multiple lung metastatic lesions. Despite intensive care, including intubation and one course of chemotherapy, the patient succumbed to the disease 43 months after diagnosis.

\section{Discussion}

DSRCT was first described in 1989 by Gerald and Rosai (8). DSRCT is a rare aggressive tumor with few long-term survivors and the prognosis of patients with DSRCT has not improved substantially since the first description of the disease. The rarity of this tumor has prevented the development of standard therapy for DSRCT. DSRCT has been reported as being associated with a characteristic reciprocal translocation $[\mathrm{t}(11 ; 22)$ (p13; q12)], which fuses the Ewing's sarcoma gene on chromosome 22 to the Wilms' tumor gene on chromosome 11 (9). This translocation is reflected by the immunohistological expression of the Wilms' tumor 1 protein in the tumor (10). The neuronspecific enolase (NSE) and CA125 proteins are tumor markers that are elevated in DSRCT patients and correlate specifically with response to treatment (11). The serum NSE levels in our case correlated well with clinical response, as shown in Fig. 3. The prolonged (15 months) progression-free survival obtained following multiagent chemotherapy suggests that DSRCT is a chemosensitive disease, as mentioned in previous studies $(1,12)$.

Efforts have been made to establish treatments aimed at controlling this disease. One of them was the combination of aggressive surgery (to resect visible disease), radiation therapy to the tumor bed and myeloablative multiagent chemotherapy (6). A recent report from the Memorial Sloan-Kettering Cancer Center experience (2) showed that overall survival in 66 patients was $44 \%$ at three years and $15 \%$ at five years using a combination of the $\mathrm{P} 6$ regimen, surgical debulking and radiotherapy to a dose of $30 \mathrm{~Gy}$. However, more than half of these patients had no distant metastasis. Currently, there is no standard therapy for patients with DSRCT, particularly for inoperable/metastatic DSRCT cases, and there are few reports of metastatic DSRCT treatment (13). To the best of our knowledge, this is the first case of metastatic DSRCT in a patient who lived for more than three years without surgical resection (14). Multi-institutional randomized control trials for DSRCT are not available due to the rarity of the disease. Another attempt at developing treatments for this disease was the use of a novel molecularly targeted therapy and a new chemical agent (15). We examined the expression of c-kit, androgen receptor, CD20 and epidermal growth factor receptor using a biopsy sample $(16,17)$. However, we found that none of the markers were expressed in tumor cells; therefore, we could not use any molecularly targeted agent in this case.

In this study, we used pirarubicin instead of doxorubicin in the P6 regimen administered to this patient, as pirarubicin may be relatively superior to doxorubicin regarding side effects (18). Moreover, the use of pirarubicin in a multiagent regimen for DSRCT was previously reported (6,7). Although our study shows only one case of treatment for metastatic DSRCT, the two modified regimens selected for our case may represent a treatment modality for DSRCT patients that has the potential advantages of decreased toxicity and improved completion rate of the chemotherapy regimen.

In conclusion, DSRCT is an aggressive but chemosensitive disease and continuous chemotherapy using an appropriate regimen with possible supportive care is essential for the long-term survival of these patients. This case report may present a treatment option for this rare disease.

\section{Acknowledgements}

We are grateful to the patient and his family for their permission to report our experience with his case.

\section{References}

1. Gerald WL, Miller HK, Battifora H, Miettinen M, Silva EG and Rosai J: Intra-abdominal desmoplastic small round-cell tumor. Report of 19 cases of a distinctive type of high-grade polyphenotypic malignancy affecting young individuals. Am J Surg Pathol 15: 499-513, 1991.

2. Lal DR, Su WT, Wolden SL, Loh KC, Modak S and LaQuaglia MP: Results of multimodal treatment for desmoplastic small round cell tumors. J Pediatr Surg 40: 251-255, 2005.

3. Gerald WL, Ladanyi M, de AlavaE, Cuatrecasas M, Kushner BH, LaQuaglia MP and Rosai J: Clinical, pathologic, and molecular spectrum of tumors associated with $\mathrm{t}(11 ; 22)(\mathrm{p} 13 ; \mathrm{q} 12)$ : desmoplastic small round-cell tumor and its variants. J Clin Oncol 16: 3028-3036, 1998.

4. Chang F: Desmoplastic small round cell tumors: cytologic, histologic, and immunohistochemical features. Arch Pathol Lab Med 130: 728-732, 2006

5. Zhang PJ, Goldblum JR, Pawel BR, Fisher C, Pasha TL and Barr FG: Immunophenotype of desmoplastic small round cell tumors as detected in cases with EWS-WT1 gene fusion product. Mod Pathol 16: 229-235, 2003.

6. Kushner BH, LaQuaglia MP, Wollner N, et al: Desmoplastic small round-cell tumor: prolonged progression-free survival with aggressive multimodality therapy. J Clin Oncol 14: 1526-1531, 1996.

7. Farhat F, Culine S, Lhommé C, et al: Desmoplastic small round cell tumors: results of a four-drug chemotherapy regimen in five adult patients. Cancer 77: 1363-1366, 1996.

8. Gerald WL and Rosai J: Case 2. Desmoplastic small cell tumor with divergent differentiation. Pediatr Pathol 9: 177-183, 1989.

9. Ladanyi M and Gerald W: Fusion of the EWS and WT1 genes in the desmoplastic small round cell tumor. Cancer Res 54: 2837-2840, 1994.

10. Hill DA, Pfeifer JD, Marley EF, Dehner LP, Humphrey PA, Zhu X and Swanson PE: WT1 staining reliably differentiates desmoplastic small round cell tumor from Ewing sarcoma/ primitive neuroectodermal tumor. An immunohistochemical and molecular diagnostic study. Am J Clin Pathol 114: 345-353, 2000.

11. Fizazi K, Farhat F, Theodore C, Rixe O, Le Cesne A, Comoy E and Le Chevalier T: Ca125 and neuron-specific enolase (NSE) as tumour markers for intra-abdominal desmoplastic small round-cell tumours. Br J Cancer 75: 76-78, 1997.

12. Al Balushi Z, Bulduc S, Mulleur C and Lallier M: Desmoplastic small round cell tumor in children: a new therapeutic approach. J Pediatr Surg 44: 949-952, 2009.

13. MrabtiH,Kaikani W, Ahbeddou N,AbahssainH,ElKhannoussiB, Amrani M and Errihani H: Metastatic desmoplastic small round cell tumor controlled by an anthracycline-based regimen: review of the role of chemotherapy. J Gastrointest Cancer: 8 February, 2011 [Epub ahead of print].

14. Miwa S, Kitamura S, Shirai T, et al: Desmoplastic small round cell tumour successfully treated with caffeine-assisted chemotherapy: a case report and review of the literature. Anticancer Res 30: 3769-3774, 2010. 
15. Thijs AM, van der Graaf WT and van Herpen CM: Temsirolimus for metastatic desmoplastic small round cell tumor. Pediatr Blood Cancer 55: 1431-1432, 2010.

16. Fine RL, Shah SS, Moulton TA, et al: Androgen and c-Kit receptors in desmoplastic small round cell tumors resistant to chemotherapy: novel targets for therapy. Cancer Chemother Pharmacol 59: 429-437, 2007.
17. Sankhala KK and Chawla SP: Review: desmoplastic small round cell tumor: current treatment approach and role of targeted therapy. Clin Adv Hematol Oncol 7: 476-478, 2009.

18. Zhai L, Guo C, Cao Y, et al: Long-term results of pirarubicin versus doxorubicin in combination chemotherapy for aggressive non-Hodgkin's lymphoma: single center, 15-year experience. Int J Hematol 91: 78-86, 2010. 